\title{
Antimicrobial Resistance Pattern of Bacteria Isolated from ICU Patients with Respiratory Tract Infections
}

\author{
Layla J afrin Soma, Mohammad Shahriar, Syeda Najah Narjish \\ and Mohiuddin Ahmed Bhuiyan
}

Department of Pharmacy, University of Asia Pacific, Dhaka, Bangladesh

Received: April 5, 2014; Accepted: October 24, 2014; Published (web): December 31, 2014

\begin{abstract}
A total of 58 samples of respiratory tract infection (RTI) patients were collected from intensive care unit of three Dhaka based hospitals namely National Institute of Diseases of the Chest and Hospital (NIDCH), City Hospital (CH) and Japan Bangladesh Friendship Hospital (JBFH). Out of 58 samples studied, 47 (81.03\%) samples showed growth of organisms identified as Staphylococcus aureus $(\mathrm{n}=24,51.06 \%)$, Pseudomonas aeruginosa $(\mathrm{n}=9$, $19.15 \%)$, Klebsiella pneumoniae $(\mathrm{n}=7,14.89 \%)$, Streptococcus pneumoniae $(\mathrm{n}=3,6.38 \%)$, Escherichia coli $(\mathrm{n}=2$, $4.26 \%)$, Citrobacter $(\mathrm{n}=1,2.13 \%)$ and Shigella $(\mathrm{n}=1,2.13 \%)$. Most of the isolates were found resistant to piperacillin/tazobactam. All the isolates of $S$. pneumoniae $(\mathrm{n}=3), P$. aeruginosa $(\mathrm{n}=9), K$. pneumoniae $(\mathrm{n}=7)$, E. coli $(\mathrm{n}=2)$, Citrobacter $(\mathrm{n}=1)$, and Shigella $(\mathrm{n}=1)$ showed $100 \%$ resistance to piperacillin. S. aureus showed $79.17 \%$ resistance to piperacillin. Most of the isolates were $100 \%$ sensitive to imipenem, whereas S. aureus was only $91.67 \%$ sensitive to it.
\end{abstract}

Key words: Antimicrobial resistance, ICU patients, respiratory tract infection

\section{INTRODUCTION}

Respiratory tract infection (RTI) has been known to be a major health problem for mortility and morbidity since many years. Still now it is one of the major causes of morbidity and mortality in the developing countries including Bangladesh. RTI is defined as any infectious disease of the upper or lower respiratory tract. Upper respiratory tract infection (URTI) includes the common cold, laryngitis, pharyngitis, tonsillitis, rhinitis, rhino sinusitis and otitis media. Lower respiratory tract infection (LRTI) includes acute bronchitis, bronchiolitis, pneumonia and tracheitis. Some of the causative agents of RTI are $P$. aeruginosa, $S$. aureus, $S$. pyogenes and K. pneumonia, E. coli, Citrobacter, H. influenza, M. tuberculosis etc. ${ }^{1}$

Correspondence to: Mohammad Shahriar

Tel: +88-02-9664953/146; Cell: +88-011-99-844-259;

E-mail: shahriar@uap-bd.edu

Dhaka Univ. J. Pharm. Sci. 13(2): 193-197, 2014 (December)
Seven deaths out of ten less than 5 years old children were reported for RTI in the developing countries. National family health survey (NFHS) revealed that two weeks before the survey $6 \%$ of under 5 children had symptoms of an RTI (cough, short and rapid breathing). Out of these children, $69 \%$ were taken to a health facility or health provider for treatment. An average adult has 2-4 episodes per year and a child has 6-8 episodes of RTI per year. ${ }^{1}$

Different antibiotics are used to treat RTI like any other infectious diseases. A study record of 150 RTI patients at a Primary Health Complex in Bangladesh from January 2009 to June 2009, showed that, the highest prescribed antibiotic was ceftriaxone $(30.19 \%)$ followed by cefixime $(18.87 \%)$, and amoxycillin $(16.98 \%)^{2}$

Antibiotics are commonly prescribed in treating common respiratory tract infections even with viral etiology. As there is difficulty in establishing bacterial etiology at the time of prescription, antibacterial therapy of RTI is usually empirical 
considering the presence of risk factors and severity of disease. ${ }^{3}$

Now a days, the inappropriate or misuse of antibiotics has become a threat worldwide because the number of antibiotic resistant bacteria is increasing day by day. These are not responding to the conventional antimicrobial agents and for that reason, physicians are looking for new antibacterial agents.

Critically ill patients admitted in intensive care units (ICUs) are always at a higher risk of developing infections with various antibiotic resistant organisms. The patients in the ICU have a 5 to 7 fold higher risk of a nosocomial infection compared to the average patient and $20-25 \%$ of all nosocomial infections develop in ICUs. ${ }^{4}$

As RTI and its outcome are very alarming, the aim of this study was to observe the severity of RTI cases among the adult and child population of our country. This research work was conducted with a view to identify the frequent organisms that are responsible for the RTI and characterize the isolated organism as per their antibiotic sensitivity and resistance pattern. Also, the objective was to find a better treatment of the infections against those organisms which have already become multidrug resistance.

\section{MATERIALS AND METHODS}

A total of 58 samples (Sputum, Pleural fluid, Pus, IT-Tube sample, Bronchial swab, Respiratory swab) of RTI patients were collected from three hospitals of Dhaka city, National Institute of Diseases of the Chest and Hospital (NIDCH), City Hospital (CH) and Japan Bangladesh Friendship Hospital (JBFH) from the period of December 2012 to March 2013. The samples were carefully and aseptically transferred to the laboratory for further examinations. The collected samples were cultured in nutrient agar media and stored at $2-8^{\circ} \mathrm{C}$ for further examinations.

Identification test of the isolated organisms. For identification of different microorganisms selective media were used in this study. For example, blood agar was used to identify Streptococci sp.,
MacConkey agar for E. coli, Klebsiella sp. and Pseudomonas sp., mannitol salt agar for Staphylococci sp. and Salmonella Shigella agar for Shigella sp.

Biochemical test. Biochemical test is necessary for specific identification of isolated microorganisms. Under this section, Catalase test, triple sugar ion agar test, citrate utilization tests, optochin susceptibility and bile solubility tests were done.

Antibiotic susceptibility test. Antibiotic susceptibility test of isolates on commonly used antibiotics were performed on Muller-Hinton agar medium by disk diffusion technique according to Clinical Laboratory Standard Institute (CLSI) guidelines (NCCLS 1997). ${ }^{5}$ Paper disks were impregnated with antibiotics such as imipenem (10 $\mu \mathrm{g})$, amikacin $(30 \mu \mathrm{g})$, piperacillin / tazobactam $(100 / 10 \mu \mathrm{g})$, ceftazidime (30 $\mu \mathrm{g})$, levofloxacin $(5 \mu \mathrm{g})$, azithromycin $(30 \mu \mathrm{g})$ and incubated at $37^{\circ} \mathrm{C}$ for 24 hours. After defined incubation period, the diameter of the zones of inhibition were measured and interpretation of result based on CLSI guideline was performed. ${ }^{5}$

\section{RESULTS AND DISCUSSION}

After evaluating the data collected from hospitals and the tests done in the laboratory, a scenario of recent antimicrobial resistance pattern of bacteria was assessed.

Out of the 58 clinical samples studied, 41 $(70.69 \%)$ were from male and $17(29.31 \%)$ were from female. Higher number of patients $(n=41$, $70.69 \%$ ) were male and among them 33 were infected ( $\mathrm{n}=33,80.49 \%)$; number of female patients $(\mathrm{n}=17,29.31 \%)$ were lower than male and 14 were found to be infected $(\mathrm{n}=14 ; 82.35 \%)$ (Table 1).

Table 1. Samples with RTI causing bacteria in two sex groups.

\begin{tabular}{lcc}
\hline Sex & No. of samples $(\%)$ & No. positive cases $(\%)$ \\
\hline Male & $41(70.69)$ & $33(80.49)$ \\
Female & $17(29.31)$ & $14(82.35)$ \\
Total & $58(100)$ & $47(81.03)$ \\
\hline
\end{tabular}


Of the 58 clinical samples studied, $47(81.03 \%)$ were collected from NIDCH, $9(15.52 \%)$ from City Hospital $(\mathrm{CH})$ Dhaka and 2 (3.45 \%) from JBFH. Figure 1 shows that highest number of patients $(\mathrm{n}=27,46.552 \%)$ belonged to age group (51-60 in years). No significant difference was found between the age groups of 40-50 and 51-60 years.

Out of 58 samples, $43(\mathrm{n}=47 ; 91.49 \%)$ samples from NIDCH and $4(n=9 ; 44.44 \%)$ samples from $\mathrm{CH}$ showed bacterial growth; no growth was found in $2(\mathrm{n}=2 ; 0 \%)$ samples from JBFH.
The common infecting organisms isolated in this study were presumptively identified as $S$. aureus $(\mathrm{n}=24,51.06 \%)$, followed by $P$. aeruginosa $(\mathrm{n}=9$, $19.15 \%)$ and $K$. pneumoniae ( $\mathrm{n}=7,14.89 \%$ ) (Figure 2). In another study carried out in Libya, Eldeeb and Khashan found that $S$. aureus $(17.71 \%$ ) was the most prevalent organism, followed by $S$. pyogenes (12.34 $\%)$ and $K$. pneumoniae $(11.27 \%) .{ }^{6}$ P. aeruginosa represented only $6.26 \%$. This present study did not find $S$. pyogens. Considering the types of infecting organisms, this study is correlated with the study of Eldeeb and Khashan. ${ }^{6}$

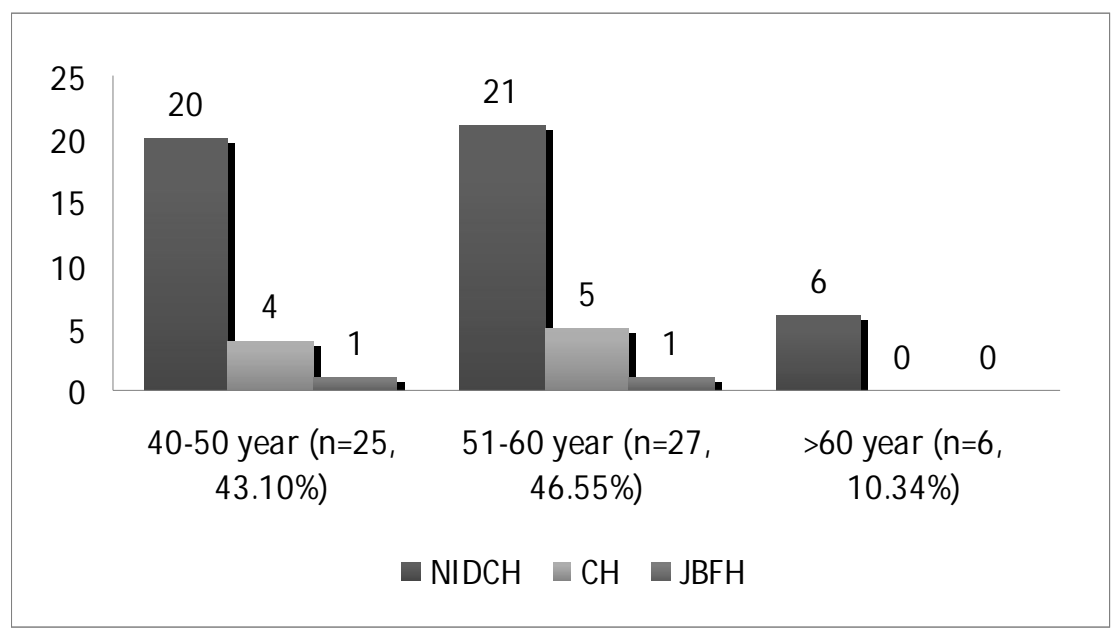

Figure 1. Age specific distribution of the study population enrolled from various hospitals.

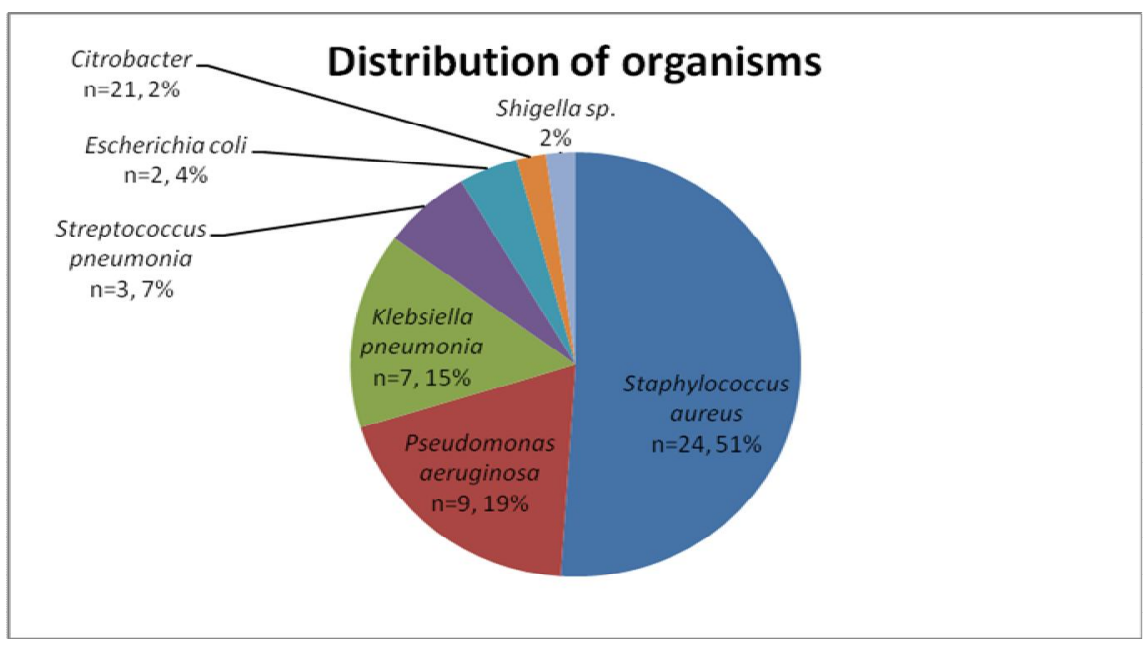

Figure 2. Distribution of organisms isolated from different samples of patients with RTI. 
Different antibiotic discs used in antibiogram (quantity/disc) are: IPM: imipenem $(10 \mu \mathrm{g})$, AK: amikacin $(30 \mu \mathrm{g})$, PI: piperacillin / tazobactam $(100 / 10 \mu \mathrm{g})$ and CAZ: ceftazidime $(30 \mu \mathrm{g})$, LE: levofloxacin $(5 \mu \mathrm{g})$, AZM: azithromycin $(30 \mu \mathrm{g})$.

Figure 3 shows the antimicrobial resistance pattern of isolated organisms. Most of the isolates were found resistant to piperacillin/tazobactam. All the samples of $S$. pneumoniae, $P$. aeruginosa, $K$. pneumoniae, E. coli, Citrobacter, and Shigella showed $100 \%$ resistance to piperacillin. S. aureus showed $79.17 \%$ resistance to piperacillin. Most of the isolates were $100 \%$ sensitive to imipenem, whereas only $S$. aureus was $91.67 \%$ sensitive to it.
A similar study was conducted among 498 admitted patients of different hospitals in Karachi. It showed that, Pseudomonas aeruginosa were isolated from $24 \%(120 / 498)$ of the lower respiratory tract patients. A higher resistance to Pseudomonas aeruginosa isolate was observed with piperacillin/tazobactam and cefipime i.e. $42 \%$ and $40 \%$ respectively. Amikacin also showed 35\% resistance. Imipenem was found to be most effective antibiotic against Pseudomonas aeruginosa $(76 \%$ sensitivity) but amikacin resistance was continuously increasing. ${ }^{7}$ This study result is very close to our findings.

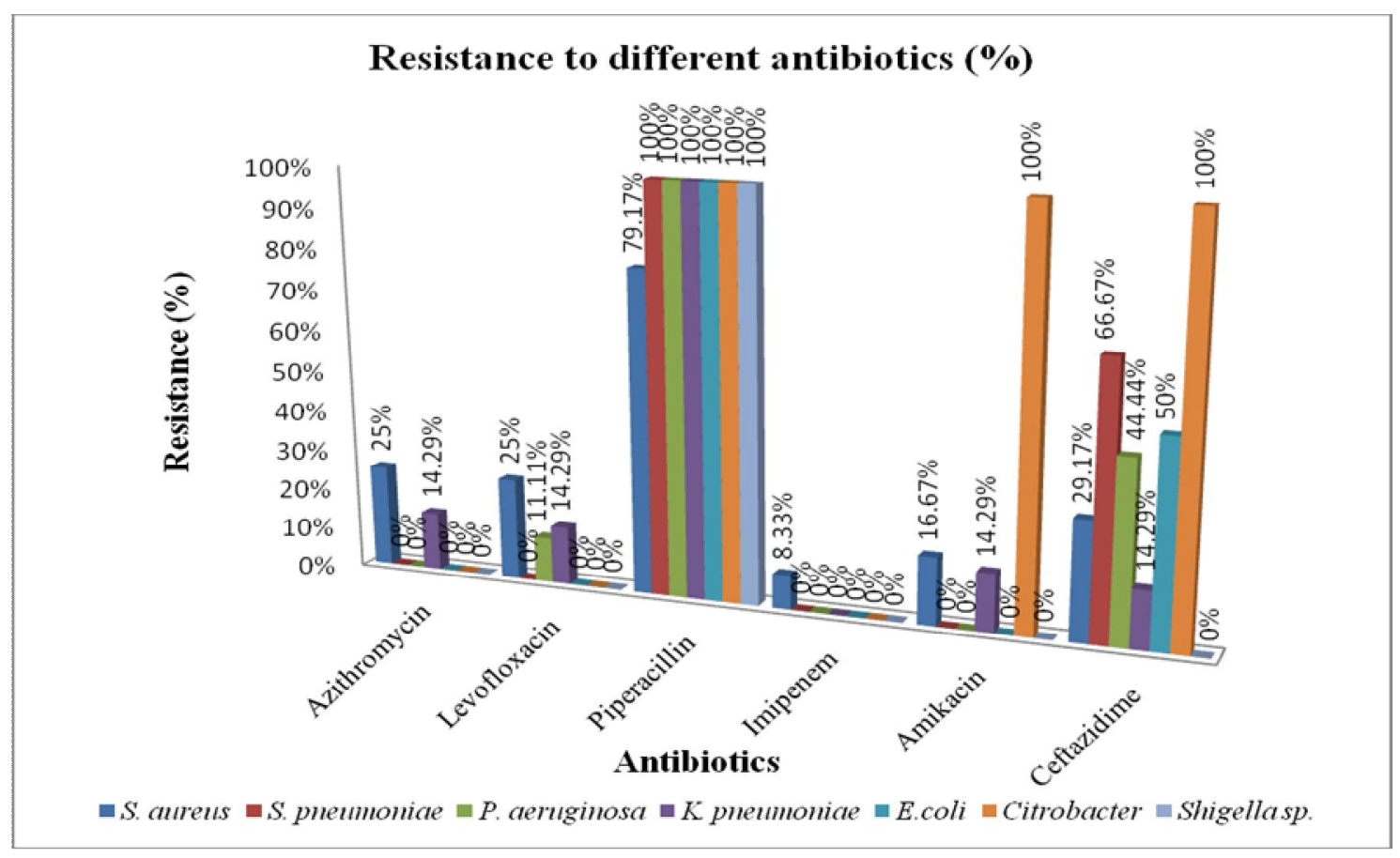

Figure 3. Antimicrobial resistance pattern of isolated respiratory pathogens.

In a published report, 42 isolates of sputum samples from different areas of Bangladesh were studied in a supranational reference laboratory (SRL) in Antwerp, Belgium. Among 42 strains, 35 (83\%) were found resistant to both isoniazid and rifampicin. Among these Multi drug-resistant (MDR) strains, $40 \%$ were found resistant to any one of the secondline drugs (kanamycin, ofloxacin, ethionamide, and para-amino salicylic acid). However, none of the strains were found to be extensively drug resistant (XDR). ${ }^{8}$

The present study along with the other studies clearly demonstrates that, day by day the UTI organisms are going to be resistant to the conventional antibiotics. Bacterial resistance is one of the major causes of failure in the treatment of infectious diseases resulting in increased morbidity, 
mortality, and costs. ${ }^{2}$ The reasons behind this ever increasing resistance could be unnecessary antibiotic prescribing even in viral infections, misuse (over use or incomplete use of a prescribed dose) of antibiotics, self prescription of antibiotics, etc. One of the study represent an important target group for efforts aimed at reducing unnecessary antibiotic use, as they receive a significant proportion of the antibiotics prescribed each year. ${ }^{9}$ Over the counter availability of antibiotics is also a reason behind this growing resistance. Proper antibacterial guideline and effective plans to manage this over prescribing tendency would be helpful for the coming years.

\section{REFERENCES}

1. Prajapati, B., Talsania, N. and Sonaliya, K.N. 2011. A study on prevalence of acute respiratory tract infections (ARI) in under five children in Urban and rural communities of Ahmedabad district, Gujarat. Nat. J. Comm. Med. 2, 255259.

2. Fahad, B.M., Matin, A., Shill, M.C. and Asish, K.D. 2010. Antibiotic usage at a primary health care unit in Bangladesh. Australian Med. J. 3, 414 - 421

3. Amin, R., Hoque, A.M.W., Khan, R.F. and Rahman, M. 2009. Considering respiratory tract infections and antimicrobial sensitivity: an exploratory analysis. Malaysian. J. Micro. 5, 109-112.
4. Bhaumik, P.V., Purav, P.G., Payal, R.N., Mitesh, P.H., Piyush, P.H. and Mahendra, V.M. 2012. Bacteriological profile and antibiogram of gram negative organisms isolated from medical and neurological intensive care unit with special reference to multi-drug resistant organisms. Nat. J. Med. Res. 2, 335.

5. National Committee for Clinical Laboratory Standards, Methods for dilution antimicrobial susceptibility tests for bacteria that grow aerobically, 3rd Edition, approved standard (NCCLS, Pennsylvania, Document M7-A3, 1997).

6. Eldeeb, A.H. and Khashan, E.M. 2006. Microbiological study on respiratory tract infections in Libya. The Egyptian. J. Hosp. Med. 24, $442-459$.

7. Anab, F., Syed, B.N., Sheikh, A.K., Shaheen, P. and Sabahat, J. 2012. Antimicrobial susceptibility pattern of clinical isolates of Pseudomonas aeruginosa isolated from patients of lower respiratory tract infections. Springer Plus 1, 70.

8. Kamal, S.M., Hossain, M.S., Islam, A.B.M.T., Begum, V., Bleumink, M.B. and Deun, A.V. Anti-TB drug resistance patterns among category 2 failure patients in Bangladesh, $1^{\text {st }}$ International Conference on Lung Health, February 2008, Dhaka, Bangladesh.

9. Sharrma, R., Chopra, V.S., Kour, G. 2009. Use of antibiotics for respiratory illness in India. J. Clin. Diag. Res. 3, 15571561. 